Check for updates

1 Department of Immunology and Inflammation

2 Department of Infectious Disease, Faculty of Medicine, Imperial College London

3 Lung Division, Royal Brompton \& Harefield Hospitals, Guy's and St Thomas' NHS Foundation Trust, London

Cite this as: $B M J 2021 ; 375: n 2843$ http://dx.doi.org/10.1136/bmj.n2843 Published: 18 November 2021

\title{
Covid-19 caseload in the UK-assessments and mitigations
}

\author{
Daniel Altmann, ${ }^{1}$ Rosemary Boyton ${ }^{2,3}$
}

It is a truism that as we approach the second anniversary of the start of the covid-19 pandemic, certainties over our management of the pandemic have become ever more elusive. This makes good policy making even more challenging and nuanced than in the early days of the pandemic, especially when healthcare priorities are counterpoised against pandemic-fatigue and the need to build back economies.

Few foresaw the planet we now inhabit, with populations differentially under threat from a spectrum of variants of concern-especially Delta with its more pernicious mix of transmission and immune evasion mutations-and variable access to and uptake across the age range of diverse vaccines. A look through any of the global dataset portals, such as the one created by John Hopkins' University, also shows very starkly the diverse state of play in different countries. ${ }^{1}$ The map of Europe, colour-coded for caseload, indicates the "dark." outlier-the countries currently faring particularly poorly, notably Romania, Ukraine, Latvia, Lithuania, Estonia, and the UK. It surely seems pertinent to pause and consider these datasets. Do they reflect events accurately or is there an ascertainment bias in the counting? And, if they do faithfully reflect the situation, what can be learnt about underlying causes?

Some argue that the UK data are unfairly skewed by the high volume of tests, especially in schoolchildren. While testing disparities can indeed skew the colouring of a simple caseload heatmap, the inescapable point is that the UK has been a European outlier for hospital admissions and deaths. The ongoing, low-level debate about tolerance levels for "living with the virus" is currently conducted against an estimated backdrop of 50 ooo excess deaths per year due to covid-19. The expectation for 21st century healthcare provision is to resist rather than tolerate early death from avoidable disease. It is a given, though rarely voiced, that living with this virus, high-volume hospital admissions, and excess deaths, come at an egregious daily cost to medical professionals, the NHS, and the population. There is a temptation to normalise these figures as being like annual flu mortality which has averaged at around 20 ooo over the past decade. However, an additional 50 ooo deaths is more in line with all annual coded mortality in the UK for ischaemic heart disease. ${ }^{2}$

Faced with these very different outcomes across Europe and elsewhere, there has been a, perhaps understandable, clamour among journalists internationally to compare and finger-point whether a given country appears, at least transiently, to be doing a little better or worse. If the roller-coaster of events since January 2020 has taught us nothing else, it is that this is a long and unpredictable battle against a formidable foe, with a need for constant vigilance and reappraisal. It is not a competition with mortality figures on the scoreboard. The analogy is a poor one, not least because the ongoing trajectory of the pandemic is so interlinked between countries and their respective measures, as evidenced by the lightning speed spread of the initial strain and then of subsequent variants across the globe.

The variables feeding into the complex equation of covid-19 caseload and mortality include a number of baseline cases, prevalent variant sequences, connectivity with other countries and populations, population demography, health status, levels of deprivation, healthcare resource allocation, specific vaccines in use, timing since vaccine rollout, vaccine uptake and coverage across the age range, stringency of and timing since lockdowns, and use of “ vaccines-plus” mitigations such as vaccine passes, masks, social-distancing, ventilation, filters, and working from home where possible. ${ }^{3}$

While there is much to be learnt from meta-analysis of successes and failures with these measures across the globe, no two countries have pursued the same course and there has been little attempt to benefit from evolving a best practice. Among countries in Western Europe, the UK has been at the lower end of the mitigation spectrum, although there are also other differences to consider. The UK was initially one of the more rapid to rollout relatively extensive vaccine coverage, using both Oxford/AstraZeneca and Pfizer vaccines, in adults. This has meant that, along with Israel, it has been among the first to experience the consequences of widescale breakthrough infections as at 5-6 months, post-vaccination antibody waning dips to sub-protective thresholds..$^{-6}$ Support for this as a key factor in Delta caseload comes from the fact that countries such as Germany, which vaccinated a little later, are now entering their own spike of cases.

Compounded with this has been the impact of being the first country outside India in which Delta expanded to become the dominant sequence. Once it was appreciated that, unlike the first wave, a key component of the Delta wave is infections within schools, many countries rapidly rolled out vaccines for teenagers. The UK did this later than elsewhere, so that children returned to school unvaccinated after the Summer holidays. Following initial policy equivocation about vaccinations in schools, uptake and speed of rollout has been disappointing. ${ }^{7}$ To this mix we must add the effect of having been early to unlock and pronounce "Freedom Day" on 19 July 2021. The Freedom Day rhetoric engendered more than an easing of restrictions. The celebratory sense of a "job done" and the chance to return to normality may have played its part in building a post-lockdown UK that is somewhat less cautious than its neighbours 
in its respect for retaining pragmatic mitigations such as masks on public transport or in schools.

The government's covid-19 strategy in the UK has focused on whether or not to adopt the more stringent level of mitigations within Plan B. From the data, it should be clear that it is a simple matter of pragmatism and survival, not of politics. Current measures are not sufficient and vaccines across the age range (including third doses), masks, greater tolerance of work-from-home, ventilation, and even use of " green pass" certification may be needed to get us safely through the winter.

Competing interests: none declared

Provenance and peer review: Commissioned, not peer reviewed

1 John Hopkins' University. https://ourworldindata.org/coronavirus

2 https://www.ons.gov.uk/peoplepopulationandcommunity/birthsdeathsandmarriages/deaths/datasets/deathsregisteredinenglandandwalesseriesdrreferencetables

3 Pagel C, McKee M. Why is England doing worse against Covid than its European neighbours? The Guardian, 7 October, 2021. https://www.theguardian.com/commentisfree/2021/oct/07/england-vaccine-just-plus-europe-covid

4 EG Levin et al. Waning immune humoral response to BNT162b2 Covid-19 vaccine over 6 months. N Engl J Med. NEJMoa2114583. doi: 10.1056/NEJMoa2114583

5 Dolgin E. COVID vaccine immunity is waning - how much does that matter? Nature https://www.nature.com/articles/d41586-021-02532-4

6 Altmann DM, Boyton RJ, Beale R. Immunity to SARS-CoV-2 variants of concern. Science 2021;371:1103-4. doi: 10.1126/science.abg7404. pmid: 33707254

7 Smyth C. Only 19\% of young teenagers in England vaccinated. https://www.thetimes.co.uk/article/only-19-of-young-teenagers-in-england-vaccinated-733lg5vm2 